\title{
Special issue on spatial econometrics in honor of Ingmar Prucha: editors' introduction
}

\author{
Badi H. Baltagi ${ }^{1}$ - Giussepe Arbia ${ }^{2}$. \\ Harry Kelejian ${ }^{3}$
}

Published online: 21 May 2018

(C) Springer-Verlag GmbH Germany, part of Springer Nature 2018

This is a Special Issue of Empirical Economics (EE) in honor of Ingmar Prucha.

Ingmar R. Prucha is Distinguished University Professor in the Department of Economics at the University of Maryland. He received his Ph.D. in mathematical economics from the University of Technology in Vienna in 1977. He has been a longstanding contributor to EE and served as an AE of the journal from 1997 to 2012. It is noteworthy that EE is a journal of the Institute for Advanced Studies in Vienna where Ingmar received a post-graduate degree in economics. The intent of this special issue is to honor Ingmar with a collection of applied and theoretical articles in econometrics with emphasis on spatial econometrics. This special issue is edited by Badi H. Baltagi, Giuseppe Arbia and Harry Kelejian.

The success of this Special Issue relies on the support of Ingmar's colleagues, friends, students and the many scholars influenced by his work. We ended up with fourteen papers passing through the rigorous review process of Empirical Economics. Of course, we would like to thank all of the referees for their hard work.

Finally, let me say that Ingmar has been a long-time friend to all of the guest editors. This special issue is a token of our appreciation to his scholarship.

Badi H. Baltagi

bbaltagi@maxwell.syr.edu

1 Syracuse, USA

2 Rome, Italy

3 Maryland, USA 


\section{Summary of contributions}

The special issue starts with a tribute to Ingmar by two of his colleagues at the University of Maryland. Kuersteiner and Chao review Ingmar Prucha's scientific contributions in econometrics and empirical economics including personal remarks regarding their colleague Ingmar at the University of Maryland.

The special issue continues with contributions to methodology as well as applications in spatial econometrics to honor Ingmar's work in this important area of econometrics.

The methodology papers include the following contributions:

Le Sage and Pace discuss Monte Carlo methodology that can be used to explore alternative approaches to estimating spatial regression models. They focus on models that include spatial lags of the dependent variable. A major point is that following publication of LeSage and Pace (2009), practitioners have relied on scalar summary measures of direct and indirect effects estimates to interpret the impact of changes in explanatory variables on the dependent variable of interest. They argue that these should be the focus of Monte Carlo experiments.

Yang introduces methods for bootstrapping the critical values of LM-type tests under non-normality and/or unknown heteroskedasticity. The outer product of martingale difference (OPMD) for estimating the variance of the score function is seen to be a crucial step in achieving robustness against unknown heteroskedasticity. Three versions of the LM-type tests and their bootstrap analogues are given for each of the two general higher-order models, namely the $\operatorname{SARAR}(p, q)$ and the $\operatorname{MESS}(p, q)$. These tests are easy to implement. Monte Carlo results show that the LM-type tests referring to the bootstrap critical values effectively remove the size distortions resulting from referring to the asymptotic critical values.

Kelejian and Piras consider spatial models with additional endogenous variables as well as nonlinearities. For these models, the authors recommend the use of nonlinear forms of the instruments. The paper provides Monte Carlo results showing gains in mean squared error when nonlinear instrumental variables are used over the standard linear instruments common in the spatial literature.

Kao and Bera discuss the problems of testing for the absence of spatial dependence in the disturbances against the alternative of spatial ARMA process incorporating the possible presence of spatial dependence in the dependent variable. They find that under the null hypothesis, the nuisance parameter is not identified, resulting in a singular information matrix (IM). Following Davies $(1977,1987)$ they propose a test procedure based on the supremum of the Rao score (RS) test statistic. Using the general test procedure of Bera and Yoon (1993) under local misspecification, they avoid the explicit estimation of the spatial autoregressive (SAR) parameter. Monte Carlo shows that the proposed tests have good finite sample properties.

Fingleton, Le Gallo and Pirotte consider a three-dimensional panel-data model with a spatial lag and a spatial moving average nested random effects disturbance. The model is estimated using a three-stage procedure. In the first step, the spatial lag panel-data model is estimated using an instrumental variable (IV) method. Using the IV residuals from the first stage, the variance components and the spatial moving average parameters are estimated using a generalized moments (GM) procedure. The third step 
applies a Cochrane-Orcutt-type procedure combined with the IV estimation. Monte Carlo results show that the performance of this three-step estimator yields similar root-mean-squared error to that of maximum likelihood. An empirical application of regional employment in the European Union illustrates this estimation method.

Handayani, Folmer, Kurnia and Notodiputro propose a spatial empirical Bayes predictor (SEBP) of the small area mean of a positively skewed variable of interest in the presence of spatial dependence among the random small area effects. Using simulations, they show that compared to its alternatives, i.e., the direct estimator which is solely based on the survey data for the small area under study, the empirical best linear unbiased predictor (EBLUP) which does not take into account spatial dependence and skewness, the empirical Bayes predictor (EBP) which takes into account skewness but not spatial dependence among the small areas, the SEBP has the smallest average relative bias and average relative root-mean-squared error for various combinations - though not all—of skewness and spatial correlation.

The applied papers include the following contributions:

Jing, Elhorst, Jacobs and Haan investigate the propagation of financial turbulence via trade, capital flows and distance channels in the pre-crisis and global financial crisis periods by modeling spillover and interdependence effects, using spatial econometric techniques. Financial turbance is proxied by the ratio of non-performing loans (NPLs) to total loans in a country. Spillover effects are defined as significant changes of the linkages between countries due to a shock, and interdependence effects as strong linkages among pairs of countries independent of shocks. Using annual data of 40 countries from 2003 to 2010, the authors find that interdependence and spillover effects should be jointly analyzed. Furthermore, their results suggest that the capital flows channel is more important than the other two channels in capturing propagation of financial turbulence. By deriving what is known in the spatial econometrics literature as direct and indirect effect estimates, they show that the marginal effects of macroeconomic variables (like GDP growth, inflation and credit growth) on financial turbulence take different forms during a crisis than in tranquil periods.

Baltagi, Egger and Kesina propose a generalized spatial panel-data probit model with spatial autocorrelation of the dependent variable, the time-invariant individual shocks and the remainder disturbances. They propose its estimation using a Bayesian Markov chain Monte Carlo procedure. Simulation results show that the proposed estimation method performs well in small- to medium-sized samples. This method is then applied to the analysis of export-market participation of 1,451 Chinese firms between 2002 and 2006 in the prefecture-level city of Wenzhou in the province of Zhejiang. Empirical results show that two of the three forms of the hypothesized spatial autocorrelation are significant, namely the spatial lag for the dependent variable and the time-invariant firm-specific shocks but not the time-variant shocks. Ignoring any of these significant spatial effects would lead to misspecification.

Basak, Bhattacharjee and Das develop a new identification result for the causal ordering of observation units in a recursive network or in a directed acyclic graph. Inferences are developed for an unknown spatial weights matrix in a spatial lag model under the assumption of recursive ordering. The performance of the methods in finite sample settings is very good. Application to data on portfolio returns produces inter- 
esting new evidences on the contemporaneous lead-lag relationships between the portfolios and generates superior predictions.

Shi and Lee study the effect of the right-to-carry laws on crime using heterogeneous time trends across US states. Using a dynamic spatial panel model with interactive effects, they find positive spatial spillovers in crime rates. An average $1 \%$ reduction in crime rates in neighboring states can decrease crime rates by $0.069 \%$ to $0.287 \%$, with property crimes exhibiting higher degrees of spatial dependence than violent crimes. They find that although the passage of right-to-carry laws has no significant effects on the overall violent or property crime rates, they lead to short-term increases in robbery and medium-term decreases in murder rates.

Baltagi, Deng and Ma study the fact that 37 percent of the internal migrants in China do not sign a labor contract with their employers, as revealed in a nationwide survey. These contract-free jobs pay lower hourly wages, require longer weekly work hours and provide less insurance or on-the-job training than regular jobs with contracts. The authors find that the co-villager networks play an important role in a migrant's decision on whether to accept such insecure and irregular jobs. By employing a comprehensive nationwide survey in 2011 in the spatial autoregressive logit model, the authors show that the common behavior of not signing contracts in the co-villager network increases the probability that a migrant accepts a contract-free job. They provide three possible explanations on how networks influence migrants' contract decisions: job referral mechanism, limited information on contract benefits and the mini labor union formed among co-villagers, which substitutes for a formal contract. In the sub-sample analysis, they also find that the effects are larger for migrants whose jobs were introduced by their co-villagers, male migrants, migrants with rural Hukou, short-term migrants and less educated migrants. The heterogeneous effects for migrants of different employer types, industries and home provinces provide policy implications.

In studies of income inequality, a fundamental distinction can be drawn between inequality of opportunity (IO) and inequality of returns to effort (IRE). Since the effects of these two components on economic growth are very different, it is of paramount importance to be able to measure them and test their significance. However, while there is already a wide literature on the measurement of the two sources of inequality, there is still a gap in the statistical inferential procedures to test the dominance of one component on the other in empirical cases. In their contribution, Arbia and Pace propose a new test for the difference between IO and IRE which is based on the ANOVA framework adapted to decompose the coefficient of variation. The proposed methodology is applied to the Italian Survey on Income and Living Condition (IT-SILC data, wave 2005 and 2011). The empirical results show that in both years father's education, region of residence and gender are important variables influencing inequality of opportunity. In addition, the role of mother's education that had a low influence on inequality of opportunity factor in 2005 has increased its influence over time and becomes significant in 2011.

Mukerji studies the impact of common investors in creating vulnerability through international spillovers in the 1980s, 1990s and 2000s. A spillover is defined as the impact on a country resulting from changes occurring abroad, where domestic economy fundamentals are assumed to remain constant. The impact arises from a purely external shock because of changes in linked countries. A vulnerability index proposed 
by Kelejian and Mukerji (2011) is used to measure the spillover. The vulnerability index describes how changes in the conditions in neighboring countries affect a given country. Neighbors refer to linked countries where the links arise due to trade and financial dealings. This vulnerability index is split into two parts: one arising from international linkages and another from prevailing domestic conditions determining sensitivity to spillovers. The empirical findings suggest a rising vulnerability index over successive decades chiefly driven by international linkages. 